\title{
The accuracy versus interpretability trade-off in fraud detection model
}

\author{
Anna Nesvijevskaia ${ }^{1,2, *}$ (D), Sophie Ouillade ${ }^{1}$, Pauline Guilmin $^{1}$ and Jean-Daniel Zucker ${ }^{3}$ \\ ${ }^{1}$ Quinten, 8 rue Vernier, Paris 75017, France \\ ${ }^{2}$ Laboratory DICEN Ile de France, Conservatoire National des Arts et Métiers, 292 rue Saint Martin, Paris 75003, France \\ ${ }^{3}$ IRD, UMMISCO, Sorbonne University, Bondy F-93143, France \\ *Corresponding author. E-mail: anna.nesvijevskaia@gmail.com
}

Received: 12 October 2020; Revised: 13 March 2021; Accepted: 22 April 2021

Key words: data analysis; fraud detection; human data mediation; interpretability; unbalanced data

\begin{abstract}
Like a hydra, fraudsters adapt and circumvent increasingly sophisticated barriers erected by public or private institutions. Among these institutions, banks must quickly take measures to avoid losses while guaranteeing the satisfaction of law-abiding customers. Facing an expanding flow of operations, effective banking relies on data analytics to support established risk control processes, but also on a better understanding of the underlying fraud mechanism. In addition, fraud being a criminal offence, the evidential aspect of the process must also be considered. These legal, operational, and strategic constraints lead to compromises on the means to be implemented for fraud management. This paper first focuses on the translation of practical questions raised in the banking industry at each step of the fraud management process into performance evaluation required to design a fraud detection model. Secondly, it considers a range of machine learning approaches that address these specificities: the imbalance between fraudulent and nonfraudulent operations, the lack of fully trusted labels, the concept-drift phenomenon, and the unavoidable trade-off between accuracy and interpretability of detection. This state-of-the-art review sheds some light on a technology race between black box machine learning models improved by post-hoc interpretation and intrinsic interpretable models boosted to gain accuracy. Finally, it discusses how concrete and promising hybrid approaches can provide pragmatic, short-term answers to banks and policy makers without swallowing up stakeholders with economical and ethical stakes in this technological race.
\end{abstract}

\section{Policy Significance Statement}

This paper discusses efforts to harness the power of information by private compagnies such as banks, which are recognized as being of public benefit to citizens and which work closely with regulatory and legal authorities on criminal acts such as fraud or money laundering. In this context, the transparency of algorithms and the interpretability of their results, pointing to suspicions of fraud, could be a critical issue: it goes beyond questions of statistical accuracy to address societal choices.

\footnotetext{
(C) Quinten SAS and Jean-Daniel Zucker, 2021. Published by Cambridge University Press. This is an Open Access article, distributed under the terms of the Creative Commons Attribution-NonCommercial-ShareAlike licence (http://creativecommons.org/licenses/by-nc-sa/4.0), which permits noncommercial re-use, distribution, and reproduction in any medium, provided the same Creative Commons licence is used to distribute the re-used or adapted article and the original article is properly cited. The written permission of Cambridge University Press must be obtained prior to any commercial use.
} 


\section{Introduction}

Fraudsters are adapting and circumventing increasingly sophisticated barriers erected in particular by banking institutions, which must take quick action to avoid losses while ensuring customer satisfaction. Faced with an increasing flow of transactions, banks' efficiency relies on data analysis to support established risk control processes, but also on understanding the underlying fraud mechanism. Furthermore, there is no escaping the search for evidence (Ryman-Tubb et al., 2018): customers with erroneously suspended transactions ask for explanations, anti-laundry authorities, such as TRACFIN in France, expect argued reporting on suspicious deposits or transfers, and legal authorities need concrete facts, in particular to distinguish between criminal and civil liability (Fraud Act, etc.). These legal, operational, strategic, and even ethical constraints lead to compromises on the means to be implemented for fraud management. This paper first focuses on the practical issues raised by fraud management and the specificities of measurement when developing fraud detection models (FDM) that address the trade-off between accuracy and interpretability of detection. Secondly, it provides a state-of-the-art (SOTA) review of the different machine learning based approaches to process these data. Finally, it examines how concrete approaches can provide pragmatic and short-term responses to banks and policy makers without forcing economically and ethically constrained stakeholders into a technological race.

\section{Fraud Detection Activities}

Fraud management process is a flow of tasks, tainted by a level of human intervention and by economic issues, specific to the nature of controlled banking operations. It cannot be reduced to a binary fraud detection automatic device, and the device must be adapted to the particularities of the process, especially in terms of metrics to optimize through an algorithmic approach. In this first part, we put in perspective the operational questions and needs in order to specify the concept of performance dear to modeling.

\subsection{Overview of fraud types in banking industry}

Fraud can be defined as all acts of cheating carried out by deceit and in bad faith for the purpose of obtaining an advantage. Fraud damages an individual, a company, an association, the State, and other. Beyond the psychological and moral impacts (Alexopoulos et al., 2007), financial losses due to fraud represent a serious risk for private and public domains, such as tax collection, insurance, telecommunications, or banking industry. This last sector is a target of choice for perpetrators as the volume of banking operations and the diversity of channels increase. Given the specificities of the banking sector and the various crises that have marked it in recent decades, the Basel commission identified fraud, and particularly external fraud, as a major operational risk in 2004. This risk then impacts the calculation of the minimum solvency ratio for banks (McDonough ratio), which is increasingly restrictive and is in the process of being standardized. Thus, the subject of fraud remains of first importance for any banking company: fraud losses are not only suffered in the immediate term but have consequences over time and are under close surveillance.

This supervision is often declined through the various banking products (credit or debit cards, cheques, private or corporate accounts, different types of loans, trading financial instruments, etc.), by type of operations (transfers, payments, direct debit, deposits, money withdrawal, etc.) or channels (physical network, ATM, paper documents, internet, mobile applications, etc.), and by fraudster's purposes (money laundering, financing of terrorism, personal or organized gang enrichment, etc.). These dimensions are strongly linked to local or national market specificities and trends, such as regulations, competition between banks and with new entrants, or customer behavior patterns in terms of bank product consumption or channel preferences. For instance, OSMP reports in 2018 that the volume of online transactions is quickly rising, and customers require near instantaneous bank response, even for international payments, whereas cheques are slowly decreasing in volume, or have even disappeared in some countries, and can be treated with a longer delay of several hours. A bank's capacity to satisfy specific customer needs and face competition are critical. 


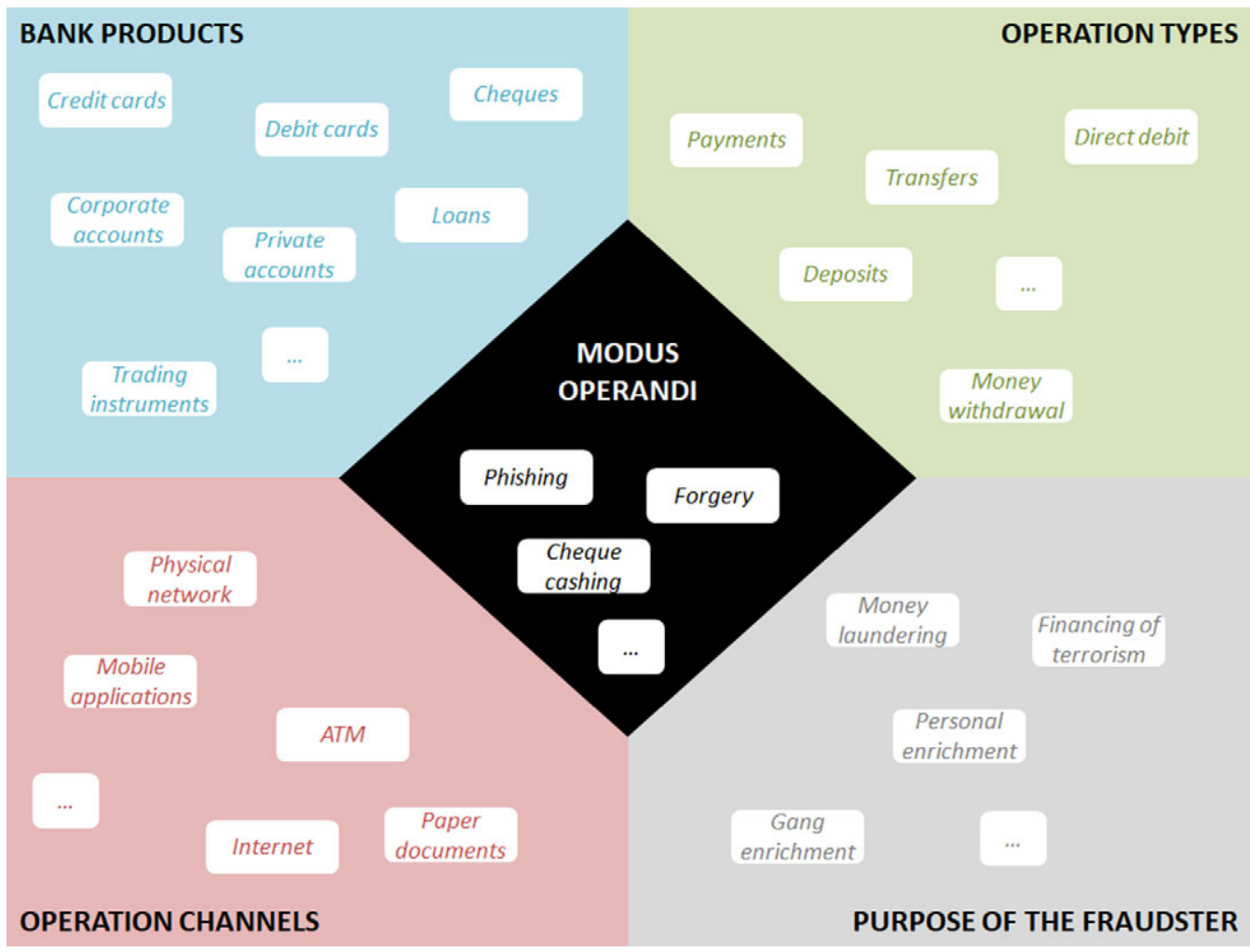

Figure 1. Illustration of fraud dimensions.

Beyond these business issues, each type of bank product, operation, channel, and fraudster's purpose can be characterized by its own data, internal (past operations, third party characteristics, internal processes or documents, etc.) or external to the bank (fraudsters behavior outside the bank or online, international blacklists, regulation and guidelines, etc.). In addition, fraud detection devices face new challenges not only in terms of growing operation volumes, but also in terms of data diversity, especially when these dimensions have to be combined. Indeed, the variety of banking services offers an increasingly wide range of opportunities for this illegal activity, and each intersection or combination of dimensions can present its own sophisticated modus operandi, including phishing, malwares, impersonation, payment theft or forgery, account takeover, and so on. The diversity of these multi-dimensional and combined tactics (see Figure 1) is enhanced with a rapid and unpredictable change: fraudsters create new approaches, test security and prevention barriers and quickly adapt. In this context, strong and dynamic fraud management is paramount for the banking industry.

\subsection{Description of different tasks in fraud management}

The global fraud management lifecycle has been described (Wilhelm, 2003) as a set of seven steps: deterrence, prevention, detection, mitigation, analysis, policy, investigation, and prosecution. With the pressure from regulators, customers, and cost reduction objectives, the mitigation step must be realized as quickly as possible without slowing down most of the usual operations. This acceleration of the process tends to break down the steps into automatable and human tasks, which can themselves be accelerated by appropriate devices. In the retail banking industry, the process can be simplified to a set of four main tasks (see Figure 2) linked to banking operations: the alerting for operations at risk of fraud, the investigation of issued alerts, the action against suspected fraudsters, and the a posteriori fraud monitoring. 


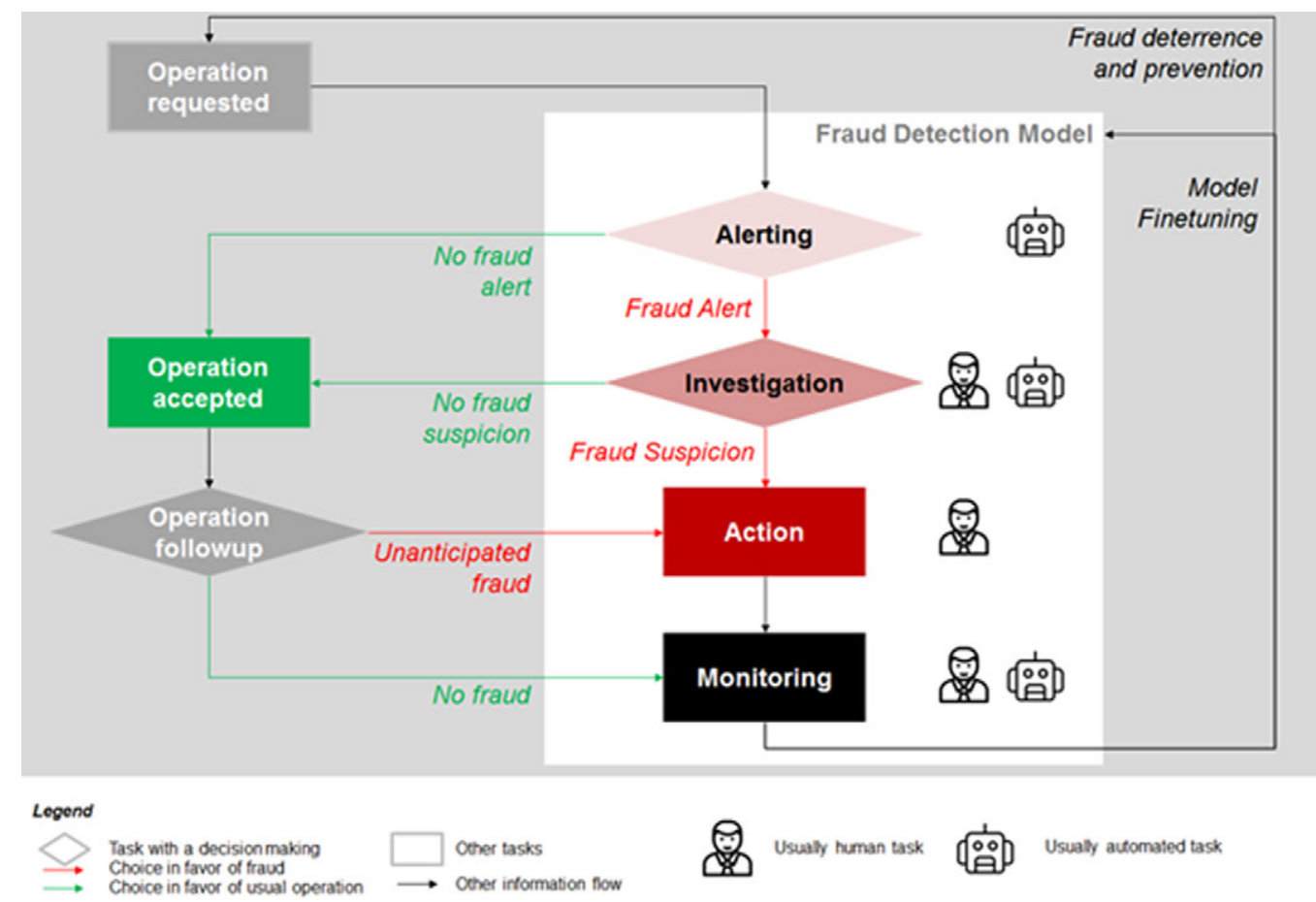

Figure 2. Simplified process of fraud management, its associated tasks and actors.

\subsubsection{Task 1: Alerting}

The first task is aimed at detecting as fast and precisely as possible a potentially fraudulent operation. Today, as most operations are too voluminous to be processed by hand, the main issue of the alerting is its automation in order to suspend or free the potential fraudulent operation without slowing down the usual operations. For most of them, such as cash withdrawal, credit card payments, check deposit, or online transfers, this task is usually automated as a set of fixed and manually adjustable business rules, and results in the suspension of the operation and the issuing of an alert. However, the accuracy of these alerts for such rare events is often insufficient: the business filters are too tight (fraud passes through the barrier) or too loose (too many operations are suspended, creating customer risk and alert treatment charge).

\subsubsection{Task 2: Investigation}

The task of investigation, often carried out manually by the back office for each alert, leads to accept the operation despite the alert, or to confirm a suspicion of fraud. The major issue of this task is the acceleration of the human investigation process: the control must concentrate on the riskier operations and quickly confirm the suspicion with explicit arguments, or, inversely, free rapidly less suspicious suspended operations. Fraud experts need for this task to have access to the right and interpretable information for each investigated operation in order to justify the suspicion and the resulting action. The task is strongly constrained by available human resources: the number of alerts that can be properly controlled is limited, and prioritization is very welcome.

\subsubsection{Task 3: Action}

Upon a suspicion, action must be taken against the potential fraudster: this third task is carried out by the bank or by the appropriate legal authority in order to quickly avoid or limit the impact of a fraud (financial loss, image, etc.). The action can also be needed following the disclosure of a fraud on a transaction that has fallen through the cracks of the previous steps: in fact, most cases of fraud are revealed once they succeeded, but the 
recorded loss can sometimes be retrieved (litigation, legal action) or recidivism prevented (customer account cancellation, etc.). The action must be ethically justified not only in terms of accusations proving fraud or a strong suspicion, but also in terms of consistency between the potential loss due to a given operation and the cost of the action and previous tasks: in this context, costly action for many low-impact frauds is not acceptable. If this task remains manual, its constraints strongly impact the previous tasks, and some subtasks can appear to be good candidates for moving up the process to accelerate mitigation and avoid manual action.

\subsubsection{Task 4: Monitoring}

The fourth and final task requires post-hoc analysis to understand the evolution of fraudsters' behavior and fraud management failures in order to better dissuade and prevent future frauds and to feed a process of continuous improvement of previous tasks: fine tuning the detection model, increasing productivity on the investigation and enhancing actions against the future fraudsters to limit the impact in terms of financial losses, moral, and image prejudice for citizens and for banks. The main issue of this monitoring task, usually remaining manual, is to be flexible enough to adjust to bank product consumption and to capture the quickly changing fraud patterns, particularly when these patterns were not detected on time.

\subsubsection{Other tasks}

The other tasks related to the FDM consist of usual operation processes as the operation request, acceptance and follow up. These tasks include physical, accounting, managerial, or legal processes, and are tainted by their own specificities in terms of paths and issues depending on the operation dimensions. The main link with the FDM is the opportunity to learn about fraudster behavior in order to better secure the operations for fraud deterrence or prevention (authentication processes, communication, regulation, etc.): this opportunity is an important alternative to the fine tuning of the FDM itself and can be reflected by other automation innovations, excluded from this paper.

\subsubsection{Process issues}

The total cost of fraud is the sum of the losses associated with criminal operations and false alerts, and investment to guard against them. This investment, technical or human, must remain lower than the expected gains, whether it is preserving margins or not losing customers. According to LexisNexis' "True Cost of Fraud Study," fraud costs in financial services from 2017 to 2018 have grown 9.3\%, which is more than the revenue growth, especially with the explosion of online and mobile channels. As the problem of task 1 can be recast as a learning problem, numerous approaches (Abdallah et al., 2016; Ryman-Tubb et al., 2018) have been developed to build models that optimize the precision of FDM to enhance or replace business filtering rules. However, the same study underlines that alerting solutions are still underperforming with too many false alerts: one-fourth of fraud costs remain dedicated to manual review, usually measured in full time equivalent (FTE) representing human intervention. In the meantime, these human tasks create the need for model interpretability in order to treat each alert or understand globally the evolution of fraudsters' behavior. In this context, minimizing false positives in the occurrence of the frauds goes hand in hand with the capacity to take into account the fraud specificities (evolving products on multiple channels, new behaviors and fraud vectors, human processes, legal and ethical issues, etc.) under profit constraint, and the use of machine learning to build the FDM raises several key questions for stakeholders and practitioners. Should the community learn the most explainable models to support tasks $2-4$ at the risk that they may be less accurate than the SOTA black box model at task 1, or should the community learn the most accurate black box models before using post-hoc methods to interpret them? Clarifying the trade-off between predictive and descriptive accuracy (Murdoch et al., 2019; Rudin, 2019) is of major importance in order to provide pragmatic, shortterm responses to the actual needs of banks and policy makers.

\subsection{Modeling fraud detection and assessing performance}

The general principle of an artificial intelligence (AI) for detecting frauds is to either detect them because their characteristics differ from that of most operations (nonfraudulent ones) or to learn a model of 
fraudulent operations from labeled operations (called examples). From an AI point of view, the first problem may be addressed using unsupervised learning or anomaly detection, the second one using supervised machine learning. But some key issues, consistent with the constraints of the bank products and tasks described above, emerge from various surveys on machine learning for fraud detection. Before describing the SOTA of the methods used to support the automation of these tasks (see next section), we first detail how the quality assessment of an AI-based FDM from a business perspective is linked to performance measure of the associated machine learning tasks, in particular regarding five main issues: the relative rarity of fraud compared to normal operations, the importance of the cost of the fraudulent operations, the difficulty of labeling operations as fraudulent with certainty, the continuously changing nature of fraud, and finally the need of interpretability.

\subsubsection{Fraud remains a "rare" event}

Among transactions, bank accounts, exchanges or any other fraud targets, most operations will be legitimate (nonfraudulent). Because fraud is so rare, datasets will be extremely biased in favor of nonfraud, which can seriously bias the models that are built from these data sets. The management of imbalanced classes generally requires data or algorithm transformation and the adjustment of precision measures. To illustrate the problem, let us consider that $3 \%$ of the transactions are fraudulent (positive). An algorithm that classifies by default all transactions as negatives (nonfraudulent) will have an accuracy of $97 \%$ although it does not detect a single fraudulent transaction. To address this issue of imbalanced data, there are various approaches. Confusion matrix, for instance, supports a more appropriate assessment of performances in an imbalanced setting. For a closer look at misclassification different metrics exist that combine the true positives (TP), false positives (FP), and false negatives (FN), ignoring the true negatives $(\mathrm{TN})$ component that drowns out fraud information. These latter are mainly based on Precision (evaluation of the ratio of correct alerts) and Recall (evaluation of the number of fraudulent observations put on alert). Among other metrics, F1-Score, Geo-Mean, or Precision-Recall curve are used to balance the trade-off between both Precision and Recall. The receiver operating characteristic (ROC) curves is also a very popular metric in the field of fraud analytics. A way to compare ROC curves is the area under the ROC curve (AUC) which computes the AUC (Davis and Goadrich, 2006). However, Precision-Recall curve and the AUC associated tends to generally better fit imbalanced data (Davis and Goadrich, 2006; Saito and Rehmsmeier, 2015).

\subsubsection{Fraud costs may vary}

Frauds account for only a small percentage of operations, and the cost of analysis of each alert can be significant, not to mention the customer risk of erroneously suspending normal operations. This leads to using metrics in machine learning that are cost-sensitive, meaning that they associate different weights to TP, FP, TN, and FN examples. However, in the case of fraud detection, the situation can be much more complex because the fraud cost includes also the financial risk of each operation. Indeed, frequent but low amount frauds, in case of a long-tailed distribution, may not be worth analyzing. Moreover, the fraud financial cost can be defined not only by the operation amount, but also by other criteria, such as a card limit reached after several low operations (Sahin et al., 2013) or a cash withdrawal amount after a fraudulent cheque deposit. Machine learning approaches rarely distinguish costs of the error according to these varying characteristics of the observations, while this aspect of misclassification can be critical to avoid drowning the signal of high impact frauds by the patterns of more frequent low impact frauds.

\subsubsection{Fraud is difficult to label}

To learn a model (be it a set of rules, or decision trees or a black box model) from examples, a dataset of examples of both fraudulent and nonfraudulent operations is required. However, because of the difficulty to assert the fraudulent status of operations, the label of the examples used in the associated machine learning task must be used with caution. Some of the operations might be labeled as "not fraudulent" although they are: the fraud will be revealed much later (see unanticipated fraud in Figure 2), or simply no 
one will ever know because the fraud has gone undetected. In machine learning, this issue of having examples that are wrongly labeled is known under the term of "noise." From a practical point of view, it means that assessing the error of classification of FDM will require some attention. When a model makes predictions on the label of unseen operations (e.g., labeling Positive the fraudulent and Negative the nonfraudulent), the confidence on both the TN and FP in the confusion matrix is clearly much lower than that of the TP and FN. It impacts the performance measure of the associated learning tasks.

\subsubsection{Fraud patterns may quickly change}

Fraud management is a fight against professionals who actively seek to subvert the system at its weakest points. They imitate the characteristics and evolution of good customer behavior to make fraudulent operations undetectable (SamanehSorournejad et al., 2016; Choi and Lee, 2018; Dhankhad et al., 2018; Achituve et al., 2019; Sadgali et al., 2019; Shah et al., 2019; Walke, 2019), and constantly test for loopholes in the fraud detection system. For the system, it means that the statistical properties of fraudulent and nonfraudulent operations evolve over a certain time. Most of the time, the system adaptation is processed manually: refinement of the rule is triggered when a specialist discovers a new type of fraud modus operandi in his bank or in a concurrent bank (change in input operations), or when he analyses the global FDM performance (global model monitoring). As legacy fraud detection systems often depend on fixed criteria or rules which hardly adapt to complex or new attack patterns (Ryman-Tubb et al., 2018), AI presents a true interest. However, for an automatic approach, particularly with a traditional supervised algorithm, recognizing old fraud behaviors that have appeared in the learning base is easy, but adapting to new behaviors as they occur is a real challenge: without retraining, the model performance declines rapidly. Furthermore, for voluminous or fast operations, such as credit card transactions, efficient automatic adaptation strategy is essential. A key question for the bank is to balance the frequency of re-training the model with its cost while considering the speed and impact of changes in both the standard and fraudulent behavior.

\subsubsection{Fraud must be explained}

After the alert is raised, the investigation task (see Figure 2) is required and a justification is needed to help the human to investigate and justify action against the fraudster. This requirement explains why FDM interpretability is expected. The quality with which a model can explain its decision is referred to as "descriptive accuracy" and although more difficult to measure than "predictive accuracy" it represents a key assessment of the quality of the FDM. There are different ways to attain a high descriptive accuracy, either building interpretable models or develop post-hoc procedure that explains decisions from black box models (Yu and Kumbier, 2020).

\section{SOTA Review of Fraud Detection}

Supervised algorithms are commonly applied to predict any given phenomenon. Although they are often applied for fraud detection as the most efficient solution, they should be used with caution in this context because of the specificities of fraud. In this section, we propose the SOTA methods that have been used in machine learning to address specifically the constraints of fraud tasks and associated performance metrics: imbalance data, lack of labeled data, concept drift, and the need for both accuracy and interpretability.

\subsection{Dealing with imbalanced data}

As seen in Section 2.3.1, one of the specificities of fraud datasets is the strong class imbalance, the "fraud" class representing sometimes less than $1 \%$ of the whole dataset. Moreover, there is an overlap of both classes (see Section 2.3.3). These two phenomena therefore make classical supervised machine learning algorithms ineffective. The clue is to give more weight to the minority class either by modifying the data itself or the algorithm used through three possibilities: under-sampling negative observations, 


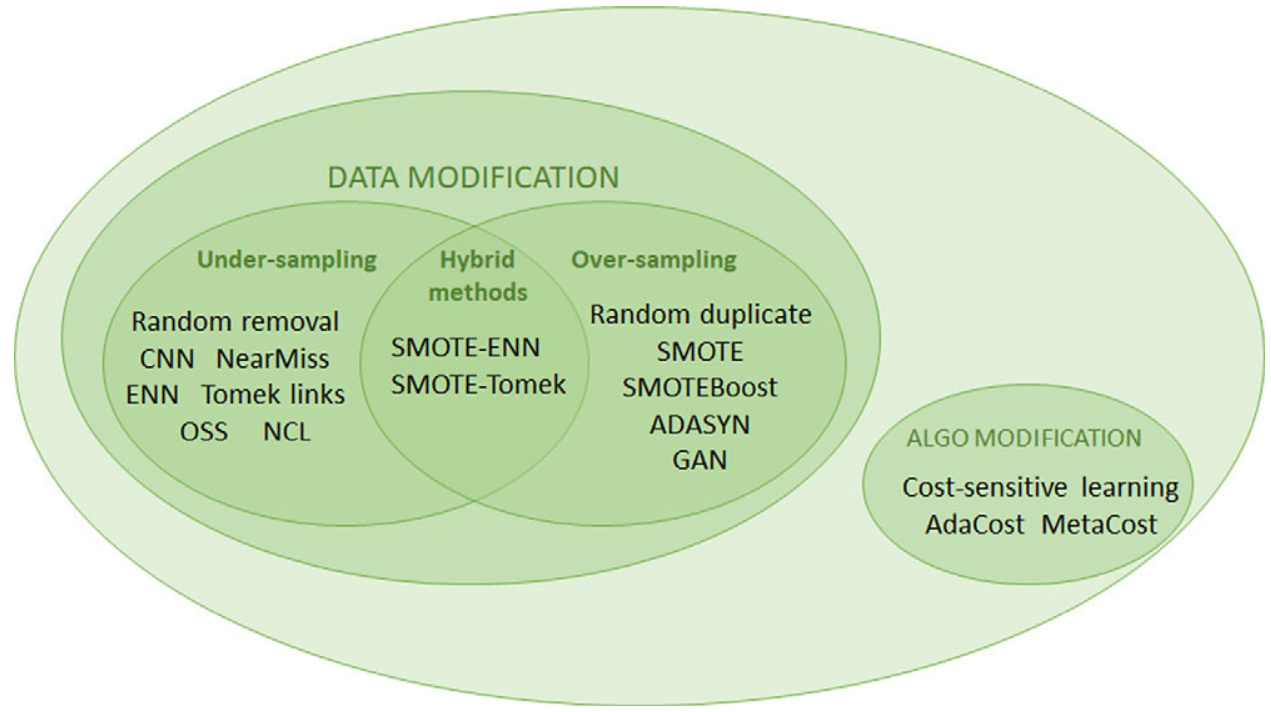

Figure 3. Summary of solutions to deal with imbalanced data.

over-sampling positive observations, or hybrid methods allowing to combine under- and over-sampling (see Figure 3 and Table A1 in the Appendix for more details).

Under-sampling consists in removing part of the observations from the majority class. The most basic method consists in removing elements of the majority class in a random way (Batista et al., 2004). Other methods try to better separate the classes to facilitate the learning of the models. This is the case of near miss and condensed nearest neighbor (CNN; Hart, 1968), selecting observations to keep. On the other hand, methods such as Tomek links (Tomek, 1976) and edited nearest neighbors (ENN; Wilson, 1972) try to select observations to remove. The latest methods combine both latter methods. For instance, one sided selection (OSS; Kubat and Matwin, 1997) mixes CNN and Tomek links. The neighborhood cleaning rule method (NCL; Laurikkala, 2001) improves OSS thanks to the ENN method. However, these methods are controversial and have poorer performance than oversampling methods (Batista et al., 2004).

Conversely, over-sampling aims to generate artificial observations of the minority class. The most basic method consists in duplicating some randomly chosen observations (Batista et al., 2004), but this method generates overfitting because of the duplicates. The synthetic minority over-sampling technique (SMOTE) method (Chawla et al., 2002) generates new observations. There are several methods which inherits from SMOTE such as BorderlineSMOTE, KMeansSMOTE, SVMSMOTE, or the well-known adaptive synthetic (ADASYN; He et al., 2008). Finally, new innovative neural networks called generative adversarial networks (GANs) have proven to be effective in over-sampling data of all kinds, including fraudulent data (Fiore et al., 2019; Shehnepoor et al., 2020). As recommended, hybridizing methods can combine both over-sampling and under-sampling, Smote-ENN and SMOTE-Tomek being two main examples (Batista et al., 2004).

On the other hand, algorithm modification consists in giving more weight to the minority. Firstly, as explained in Section 2.3, it is crucial to adapt performance metrics in order to optimize parameters and assess the validity of the algorithm considering the imbalance of the class. Furthermore, some algorithms (mainly tree structures) take into account the imbalance of the classes as, for instance, SMOTE Boost (Chawla et al., 2003), MetaCost (Domingos, 1999), and AdaCost (Fan et al., 1999). Another approach is to give more weight to fraudulent observations than to normal observations. This changes the cost function and it is named cost-sensitive learning (Elkan, 2001). The cost of misclassifying fraudulent observations is here globally modified. Hence it cannot respond to the issue of long-tailed costs (see Section 2.3.2) linked to the amount of the operation (local modification of the cost), should it be simple or combined to other factors. One possible solution is to under-sample the dataset thanks to business 


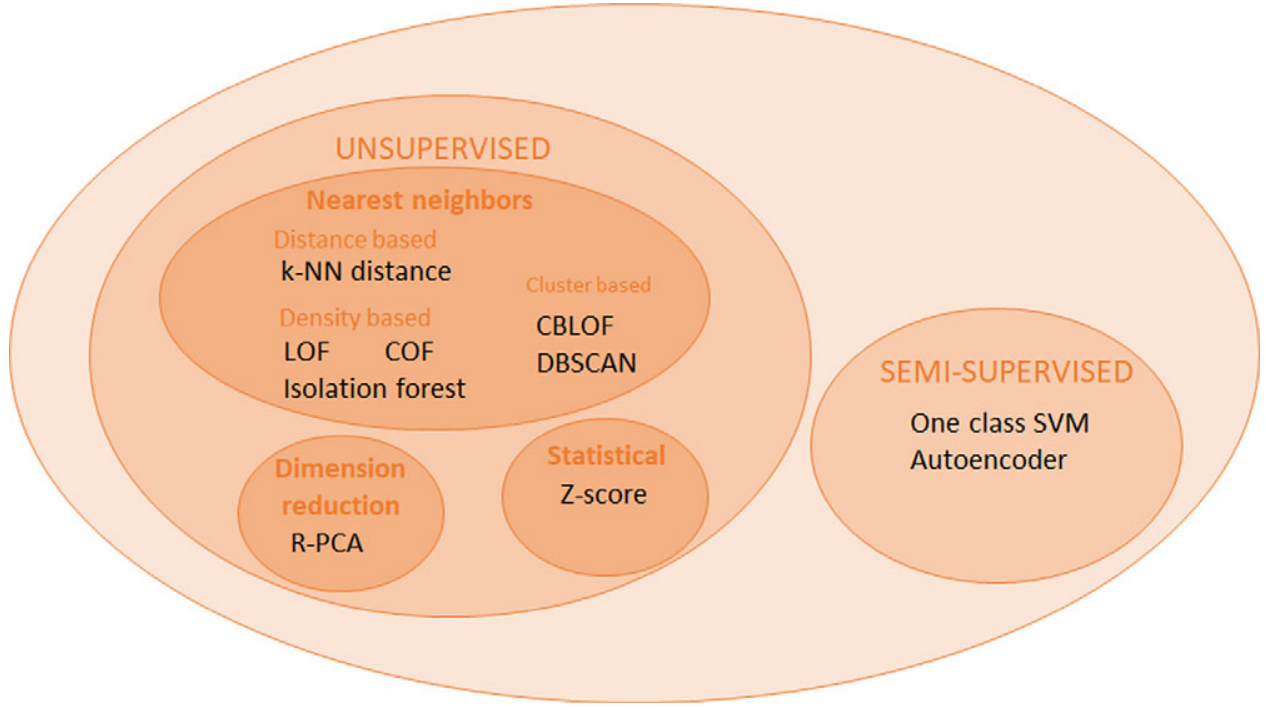

Figure 4. Summary of methods used for anomaly detection.

knowledge, such as: "In general, isolated fraudulent contactless payments of less than $10 €$ are not worth treating." This business under-sampling allows us to only focus detection on costly frauds.

When dealing with fraud data, using data level balancing techniques, like SMOTE, tends to be preferred instead of modifying the algorithm (Brennan, 2012; Dal Pozzolo et al., 2014).

\subsection{Dealing with the lack of fully trusted labels}

Beyond the limits of supervised models on imbalance data, they prove to be even less efficient on poorly labeled phenomena. Indeed, a characteristic of successful fraud is that it has gone unnoticed in history. This means that real fraud datasets often have frauds among the "nonfraudulent" observations. It is therefore recommended, in the case of not fully trusted labeled data, to use anomaly detection methods that are semi-supervised or unsupervised. Figure 4 presents the main algorithms (see full description in Table A2 in the Appendix).

Semi-supervised algorithms are trained on nonfraudulent observations and then detect abnormal, and therefore potentially fraudulent observations, during the test phase. Among them, the best known is Oneclass SVM (Schölkopf et al., 2001). With the advent of neural networks, many neural approaches to semisupervised learning, such as models based on auto-encoders (Hawkins et al., 2002; Aggarwal, 2017) have been developed for fraud detection.

Several categories of unsupervised methods coexist. Most methods are linked to the neighborhood of the observations, and can be distance-based, such as k-nearest neighbors (k-NN; Knorr and Ng, 1998; Ramaswamy et al., 2000), or density-based, attempting to identify isolated points as anomalies. Among density-based methods, local outlier factor (LOF; Breunig et al., 2000) is a local method which was improved to connectivity-based outlier factor (COF; Tang et al., 2002), and isolation forest (Liu et al., 2012) uses tree algorithms. Most recent methods are based on other clustering methods aiming to detect anomalies, such as cluster-based local outlier factor (CBLOF; He et al., 2003) or density-based spatial clustering of applications with noise (DBSCAN) algorithm (Campello et al., 2015). Finally, some wellknown categories of unsupervised methods are also used for anomaly detection, including former methods using statistical tests (Rousseeuw and Driessen, 1999) or the robust principal component analysis (r-PCA; Candès et al., 2011), based on dimension reduction.

According to surveys (Kou et al., 2004), anomaly or outlier detection was one of the main techniques used in credit card fraud detection in 2004 and it was still the case in 2016 (Abdallah et al., 2016). This can 
be explained by the fact that this kind of algorithm can easily handle not only the lack of trusted labels, but also partly answer to the problem of constant fraud evolution: a so-called concept drift problem.

\subsection{Dealing with the drifting of frauds}

As fraudsters adapt their behavior according to the product and control techniques evolution, the fraud patterns change: this phenomenon, called concept drift, appears when the underlying distribution of target concept depends on hidden contexts (Widmer and Kubat, 1994), and results in the need to re-train the model.

Besides anomaly detection described above and manual adaptations through operations or model analysis, seen in Section 2.3.4, partly dealing with the phenomenon, the classifier in supervised models can be continuously updated when new fraudulent operations become available, for example through regular relearning on a sliding window (Dal Pozzolo et al., 2015). For gradually drifting environments, such as bank cheque operations, it is possible then to choose a specific frequency or threshold to re-train the model. It is named offline learning mode (Gama et al., 2014). However, in contexts of risk of abrupt changes or voluminous and swift operations, such as for credit card transactions, new stakes arise adapt to concept drift, be robust to noise and able to treat recurring contexts (Tsymbal, 2004). It is known as stream data and it involves developing online learning solutions (Gama et al., 2014). All the above re-learning modes are illustrated in Table 1 before developing this last technique.

The easiest way to deal with online learning is to process incremental models. However, those methods, such as Hoeffding trees (Domingos and Hulten, 2000), require adapting the model for each new example. In order to use less calculation memory and time, several methods propose to work on a window containing the most recent examples, of fixed or adaptive size, as in FLORA2 (Widmer and Kubat, 1994).

To decide whether to learn the model again or not, it is necessary to detect drifts. A lot of statistical tests consist in comparing the statistical distributions such as cumulative sum (CUSUM) (Bissell, 1986) inspired by sequential probability ratio test (SPRT) or former methods such as Shiryae-Roberts test (Shiryaev, 1961; Roberts, 1966). More recent models tend to adapt the size of window to compare distributions, ADWIN is one of these examples (Bifet and Gavaldà, 2007). Afterwards, when the concept drift has been detected, there are several possibilities: learn again the model on complete historical data or on new observations, or use an old context model as in FLORA3 (Widmer and Kubat, 1994).

This subject is at stake as more and more areas requisite to work with stream data, and several solutions combining online learning and drift detection have been generally developed (Žliobaite, 2010; Gama et al., 2014; Webb et al., 2016; Žliobaite et al., 2016; Lu et al., 2019) and adapted to fraud detection (Dal Pozzolo et al., 2014; Dal Pozzolo et al., 2015).

\subsection{Dealing with the need of interpretability}

As the three latter parts deal with analytical constraints, the last operational requirement, described in Section 2.3.5, is still unresolved: practitioners need interpretable results to act. We identify three main approaches in this direction, as shown in Figure 5: intrinsically interpretable models and post hoc specific or agnostic methods.

Table 1. Procedures addressing the concept drift issues in fraud detection.

Re-training mode

\begin{tabular}{lll}
\hline Operator & Analyzing input operations & Monitoring globally model outcomes \\
\hline $\begin{array}{c}\text { After a rare fraud event: new fraud behavior } \\
\text { detected by expert teams }\end{array}$ & $\begin{array}{c}\text { Decrease of global performances essentially } \\
\text { seen through the reporting }\end{array}$ \\
& $\begin{array}{c}\text { Change of distribution of one(s) variables } \\
\text { detected } \\
\text { Online learning }\end{array}$ & $\begin{array}{c}\text { Re-training planned on a certain frequency or } \\
\text { at a certain threshold } \\
\text { Offline learning }\end{array}$ \\
\hline
\end{tabular}




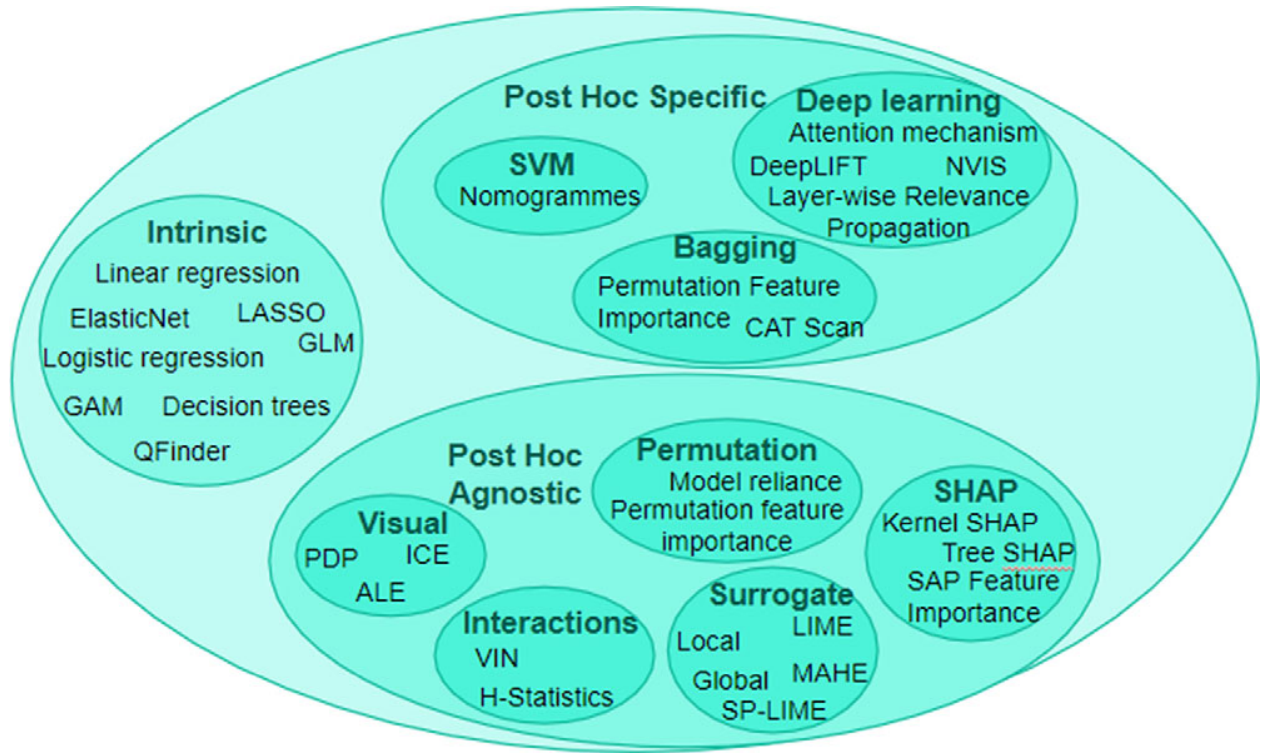

Figure 5. Summary of approaches addressing interpretability issues.

Intrinsically interpretable models, such as linear regression, logistic regression, decision trees, general additive models, or combinations of business decision rules, are characterized by their transparency and by a self-explainable structure. They are generally applied for use cases with legal or policy constraints (Zhuang et al., 2020), but they may well be not accurate enough for tasks such as fraud detection, which have high financial stakes. This explains why more accurate black box models look appealing as soon as a post hoc interpretability method is applied to provide explanations on either how they work or on their results.

Among these methods, some, called post-hoc specific, are specific to a type of model. Examples of such methods when dealing with sets of decision trees are the classification aggregation tablet scan (CAT scan) (Rao and Potts, 1997) or the feature importance permutation metric (Breiman, 2001). For support vector machines (SVM), nomograms (Jakulin et al., 2005) were a new visualization approach. Finally, for neural networks, known for better performances on some problems but also for their interpretation complexity, several specific techniques have been developed such as deep learning important features (DeepLIFT; Shrikumar et al., 2017) or layer-wise relevance propagation (Bach et al., 2015). The main disadvantage of the latter is that their use is restricted to a single type of model and it is therefore complicated to compare performances and explanations of several different models.

To counter this disadvantage, post-hoc model-agnostic methods can be used. They can be macro (or global) in order to obtain an overall view of the model to understand it in its entirety, or finer (or local) to study a particular case or observation.

As visualization is one of the most useful tools for interpreting models, visual interpretation approaches have been implemented. The main ones are partial dependence plot (PDP) curves (Friedman, 2001) and accumulated local effect plots (ALE) curves (Apley and Zhu, 2019) for macro approaches and individual conditional expectation curves (ICE; Goldstein et al., 2014) for local approaches. These methods reveal the effect of a variable within a model. In order to better choose which variables to plot, it is necessary to know which variables have the greatest influence on the prediction and are therefore the most important for the model. For this, the model reliance measure (Fisher et al., 2019) is the agnostic version of permutation feature importance measure (Breiman, 2001). Moreover, if variables interact with each other in a model, visualizing their effects separately is not enough. Methods, such as interaction strength (Friedman and Popescu, 2008) or variable interaction networks (VIN; Hooker, 2004) allow to analyze and interpret the effects of interactions between variables. 
Another classic interpretation technique is to use, after a black box model, another more interpretable model. These are the surrogate models, also known as approximation models or metamodels. The bestknown local surrogate models are local interpretable model-agnostic explanation (LIME; Ribeiro et al., 2016), model-agnostic hierarchical explanations (MAHE; Tsang et al., 2018), and Shapley additive explanations (SHAP; Lundberg and Lee, 2017) as well as all its derivatives: KernelSHAP, SHAP features importance, SHAP summary plot, and SHAP dependence plots (Lundberg et al., 2019). There are also methods based on counter-factual reasoning (Bottou et al., 2013): "counterfactual examples" that have been shown to be useful (Wachter et al., 2018) and can be easily understood, directly facilitate decisions for a user. They aim at answering the question "how is the prediction modified when the observation changes?" Finally, let us mention the knowledge distillation approach a process that consist in transferring knowledge from a large model (e.g., a deep learning one) to a smaller one, more interpretable (e.g., a decision tree; Hinton et al., 2015).

In the context of fraud detection, some authors believe that it is necessary to strengthen interpretable models to increase accuracy (Rudin, 2019), while others prefer to use advanced black box models and then apply post-hoc interpretability methods (Weerts et al., 2019). This opposition brings us to the last issue related to dealing with fraud tasks.

\subsection{Dealing with accuracy under constrains}

Fraud detection is characterized by financial and operational constraints requiring both precision, and interpretability of the results of the models. This trade-off between precision and interpretability is a key issue in the field of machine learning (Shukla and Tripathi, 2011), where both concepts are considered as "contradictory." In particular, the most recent techniques such as deep neural networks, are facing "the barrier of explainability" (Arrieta et al., 2020). The opposition between accuracy and interpretability appears in several application fields (Yang and Bang, 2019), and the performance of the most representative models is subject to comparative tests (Sahin, 2020), even if these tests are still being debated (Morde, 2019). Indeed, as mentioned in the introduction of this SOTA review, in many cases, traditional supervised models outperform other combinations of methods designed to overcome the specificities of fraud. For instance, random forest (a boosting method based on decision trees), or XGBoost (a gradient boosting type of method), adding layers of boosting to this structure by giving more weight to poorly learned observations, often performs better on fraud data. This trade-off is often schematized as a ranking of the main models along two axes: one accounting for accuracy ( $x$-axis) and the other for interpretability ( $y$-axis; see Figure 6, inspired by the above papers).

The improvement directions for basic models in Figure 6 illustrate the race between different machine learning tribes (Domingos, 2015), where advocates of black box models (such as deep learning, from the tribe of Connectionnists) try to gain interpretability (yellow arrows), while advocates of interpretable models (Symbolists, Bayesians and Analogyzers), try to gain precision (blue arrows). Only most advanced researchers in both tribes have recently started to call for hybrid methods development (Fridman, 2019). In addition to this possible future reconciliation in the field of machine learning, the comparison between the first generations of automated systems in industry, mainly rule-based, and recent approaches to machine learning have begun to highlight the need for interdisciplinary research in order to better integrate human factors considerations (Lau et al., 2018). In the next section, we present a concrete industrial application to deploy FDM in which all constraints mentioned above, including human factors, are considered.

\section{Experimentation}

The SOTA shed some light on a race for the best technology between black box machine learning models improved by post-hoc interpretation and intrinsic interpretable models boosted to gain accuracy, and the mains analytical streams to deal with other specificities of fraud detection. In this section, we concentrate on a real-world application to experimentally illustrate the differences between these two approaches under operational constraints. 

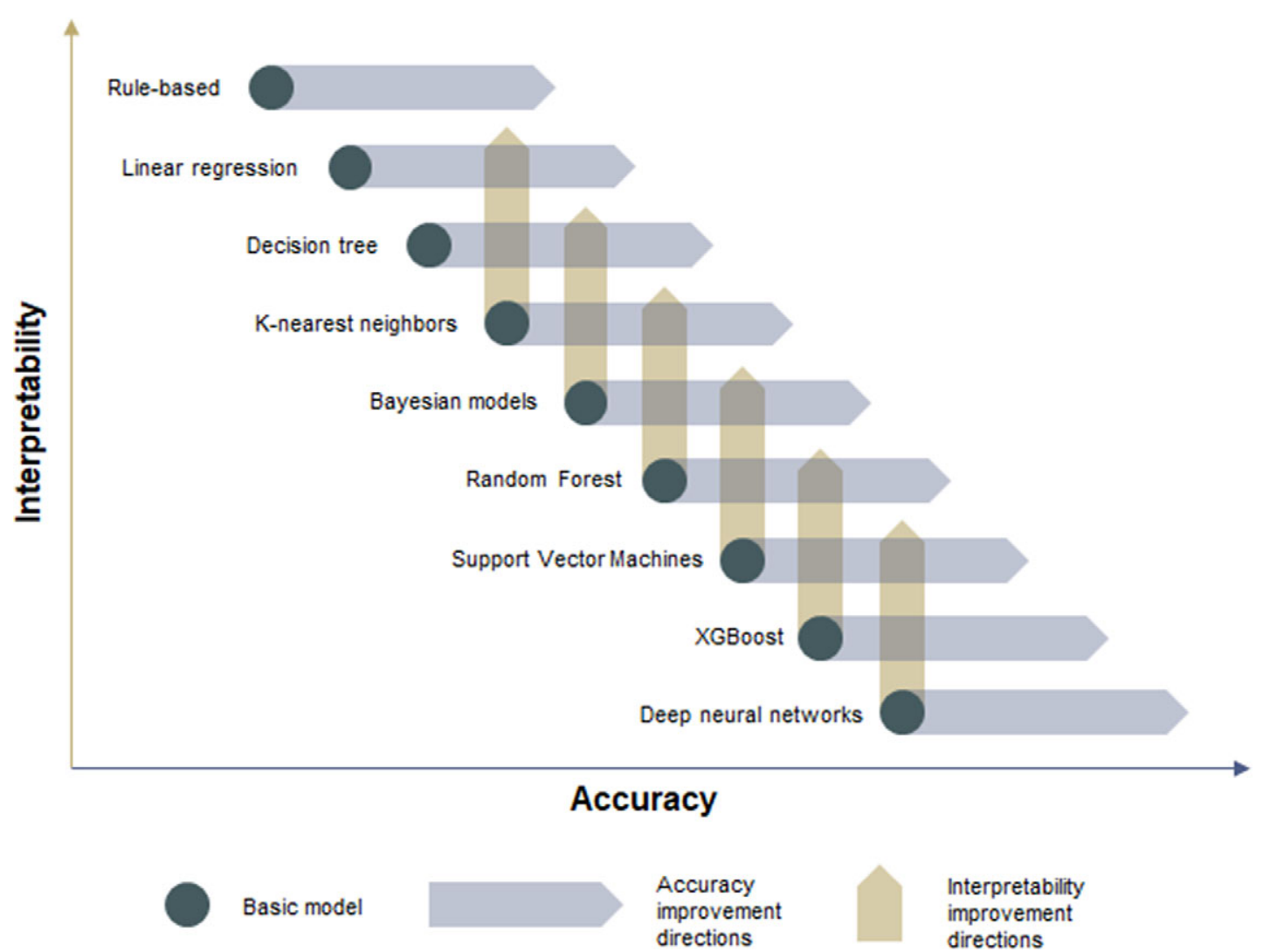
Interpretability directions

Figure 6. Interpretability vs accuracy trade-off: main models and their improvement directions.

\subsection{Experimentation context}

The experimentation takes place in the framework of a European project financed by the ERDF, called Cerberus, where two private AI actors had to cooperate to propose innovative solutions for fraud management. The project resulted in the deployment of two solutions, closely related to their application characteristics.

First, an anti-fraud software, carried by the publisher Bleckwen, is developed for instant cash transfer fraud, characterized by high operation frequencies and limited human involvement. This software is based on the improvement of a black box scoring model (XGBoost), resulting in a fraud probability score, completed with a local interpretative overlay: all operations over a given optimal threshold are suspended and must be investigated.

The second solution is an adaptation of the intrinsic interpretable analysis methods based on the Q-Finder algorithm, with proven results in medicine (Amrane et al., 2015; Zhou et al., 2019), to the world of fraud by consulting firm Quinten. This method generates automatically from historical data optimized business decision rules, as limited combinations of patterns, and classifies the new operations into two categories (fraud or not) as a binary alert. It allows to optimize cheque fraud management, which is characterized by lower volumes and slower human processes (instant operation is not needed as customers accept a several hours delay for this operation). In this second context, an operational combination of both of models (rules generation and fraud scoring) is implemented to explore the benefit of a hybrid approach of the FDM. In the following section, we present the detailed description of this second solution, including the performance achieved and the deployment issues of this promising hybridization. 


\subsection{Description of the experimentation}

The experiment on cheque fraud management followed six main steps, typical of data mining processes such as CRISP_DM (Shearer, 2000; Wirth and Hipp, 2000).

\subsubsection{Business understanding}

The constraint of interpretability emerged quickly in the first step of experimentation. Besides the need to justify action (account suspension, declaration of suspicion to authorities, etc.) and to help human investigation (experts analyze easily a limited number of fraud factors), the process of cheque fraud detection implied different human teams at each step: these teams had to collaborate and share a common understanding of the fraud suspicion factors. The exact business rules applied on the first automated alerting task were known only by three persons: these domain experts modified manually the alert criteria according to their perception of fraud drifting and kept the rules confidential in order to avoid leaks to fraudsters. The process was already operational, with an anti-fraud department able to handle no more than 30 daily alerts with the existing FTE. However, the business rules were still not precise enough: the frauds represented several millions of euros lost per year. The aim of the experimentation was then to optimize the business rules under human resource constraints and fluidify the entire process, from alerting to monitoring.

\subsubsection{Data understanding}

The data collected for the experimentation included the customer characteristics, the cheque deposit characteristics and their dynamic, the other main transactions dynamics, the historical frauds and several other confidential data sources. The collected data represented 85 tables from 5 different sources and covered the period from October 2018 à September 2019. This represented over 16,000,000 historical cheques. All the collected variables, that is more than 200, were clarified in terms of meaning and structure, and qualified following the documentation framework Databook (Nesvijevskaia, 2021). After this qualification work, the scope of the data was filtered to only those cheques that were usable and suitable for the analyses.

\subsubsection{Data preparation}

The data sources were iteratively and collaboratively structured into a matrix through feature engineering representing 343,609 historical cheque deposits characterized by 300 patterns calculated from the source variables. Only 130 of these patterns were considered relevant after discussions with the business experts and removal of redundancies. These patterns were used in the modeling phase and classified into 12 categories to facilitate the interpretation of the results. Historical frauds with confirmed financial impacts were manually reconstructed for the analyses. Moreover, all operations under a certain businessdefined amount were excluded at this preprocessing stage as not worth investigating: this treatment avoids drawing the characteristics of heavy frauds by those of frequent frauds, and, simultaneously, generates an under-sampling of nonfraudulent operations. The rare fraud phenomenon represented $0.1 \%$ of the cheque deposits. The final learning matrix was split into 9 months of historical data to train the models and 3 months to have a test set. The train test was divided into three folds stratified on fraudulent clients in order to optimize random forest model.

\subsubsection{Modeling}

The hybrid method targets the completing or replacement of business rules by optimized rules, and the combination of this optimized rules with an online-learning scoring.

For this, Quinten's method using the Q-Finder algorithm generates many intersected rules to detect the phenomenon of costly fraud. The advantages are that the fraud coverage by the rules is very good and that the variables involved in each rule are co-constructed through features engineering with the business experts to ensure easy interpretability. However, at this stage the approach generates a set of rules with too many false positives, which can lead to customer dissatisfaction and to an inability to deal with all the alerts by the anti-fraud department. Thus, an optimal combination of rules is created in order to reduce the 
Table 2. Confusion matrix on the test set.

\begin{tabular}{lll}
\hline & Positive & Negative \\
\hline True & 56 & 58,084 \\
False & 940 & 16 \\
\hline
\end{tabular}

number of false positives and maintain good coverage: for this, an F-beta score is used with, for example, beta equal to 3 to give more weight to the Recall.

This rule generation model is hybridized in a second step with a black box score model developed in parallel on all the operations. This scoring model is intended to be applied after the rules, so that all alerts can be prioritized by the score. This prioritization speeds up the work of the fraud experts without deteriorating the intelligible explanation of the fraud. If the number of alerts punctually creates an investigative workload that is even less than the capacity of the dedicated human resources, the set of rules can be completed with an additional rule corresponding to the operations with a score over a very high threshold: in this case, only this part of alerts, as a complementary "scoring rule," is harder to interpret, but the coverage of the fraud is enhanced for available human resources.

At this stage, the statistical assessment of the accuracy of the model was a Precision of $5.6 \%$ and a Recall of $77.8 \%$ on the test set, calculated on the basis of the confusion matrix presented in Table 2 .

Furthermore, as one of the main characteristics of fraud is the concept drift, the rules may no longer be accurate after a certain time of changes in fraudsters behavior. To get around this problem, the black box model is enhanced with online learning, thus the addition of a rule based on the score model threshold maintains the efficiency of the FDM by detecting new fraudster behaviors. Most of the operations in alert will then have an explanation given by the business rules, the others will correspond to new fraud techniques not learned yet by the rules. These alerts will require more investigation time from the fraud experts because they will not have a business explanation that can be quickly understood. When the number of alerts not explained by the business rules starts to be too high, new optimized rules must be learned from on the recent data: this statistical-economic equilibrium is closely followed as part of the monitoring task. A new business investigation will help to understand where these new frauds come from thanks to the new rules created, optimize fraud deterrence and prevention if possible, and finetune the FDM. During all the lifecycle, only exceptional frauds would have passed under the radar: these outliers did not appear to be economically pertinent to detect. At this stage, the articulation of the optimized rules and the online-learning score is ready for business evaluation.

\subsubsection{Evaluation}

As the decision to deploy the solution is based more on business performance indicators than on statistical ones, here are some synthetic results obtained using data from Quinten's partner about cheque fraud. For reasons of confidentiality, only the numbers of fraud cases detected will be reported, although the amounts were part of the analysis. On Figure 7, we can see that most of the fraudulent operations detected each month by a score with a threshold at $50 \%$ are also detected by the rules, so the use of interpretable models can be easily privileged for operational reasons: prioritize by the scoring model and explained by the rules. Moreover, the combination of the two models gives better results while adding only a limited number of daily alerts, as shown on Figure 8. In this example, the last month's frauds are not observed for the moment: the rules are still functional alone.

This hybridization thus answers key issues defined during the business understanding and was judged as immediately operational and very promising by the first banking partners.

\subsubsection{Deployment}

The solution, covering all the tasks of fraud management mentioned in Section 2.2 and resumed in Table 3, is being deployed in association with two user interfaces designed for the collaborative 


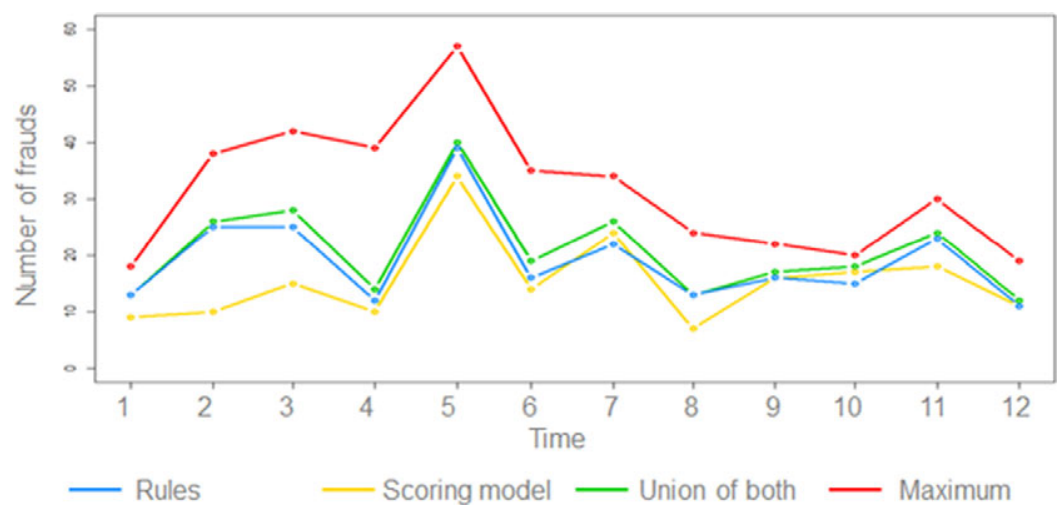

Figure 7. Number of true fraud cases detected over 1 year.

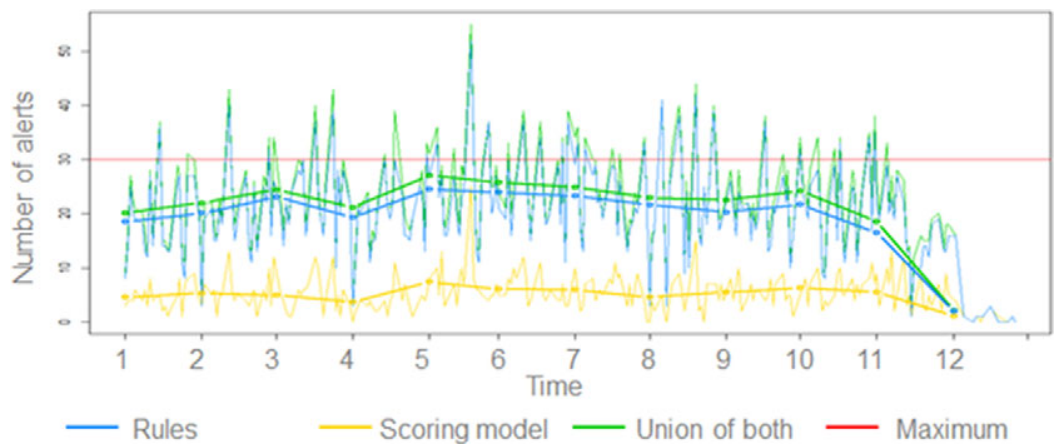

Figure 8. Average number of alerts per day over 1 year.

Table 3. Synthesis of the answers of the hybrid fraud detection model (FDM) to fraud management task issues.

\begin{tabular}{|c|c|c|}
\hline Tasks & Rules & Score \\
\hline Task 1: Alerting & $\begin{array}{l}\text { Statistically optimized set of business } \\
\text { rules for automatized alerting }\end{array}$ & $\begin{array}{l}\text { "Scoring rule" completing the set } \\
\text { without overcharging the number } \\
\text { of alerts }\end{array}$ \\
\hline Task 2: Investigation & $\begin{array}{l}\text { Interpretability of each alert through a } \\
\text { combination of explicit fraud patterns } \\
\text { for each rule }\end{array}$ & $\begin{array}{l}\text { Prioritization of alerts by fraud } \\
\text { score in order to gain in } \\
\text { investigation productivity }\end{array}$ \\
\hline \multirow[t]{2}{*}{ Task 3: Action } & \multicolumn{2}{|c|}{$\begin{array}{l}\text { Concentration of the model on heavy frauds only. Concession to get through } \\
\text { exceptional frauds that are too costly to deal with }\end{array}$} \\
\hline & $\begin{array}{l}\text { Justification for action facilitated by } \\
\text { interpretability }\end{array}$ & $\begin{array}{l}\text { Acceleration for the more and the } \\
\text { less suspected alerts }\end{array}$ \\
\hline Task 4: Monitoring & $\begin{array}{l}\text { Generation of knowledge on new } \\
\text { fraudsters' behaviors and, when } \\
\text { needed, of optimized rules }\end{array}$ & $\begin{array}{l}\text { Online-learning model adapting to } \\
\text { the fraudsters' behavior }\end{array}$ \\
\hline
\end{tabular}


investigation and the monitoring tasks. If further model optimization seems tempting, such as the interpretative overlaying model on the "score rule" alerts or a complementary outlier model for new fraud trends, other considerations appear as much more pragmatic, such as fraud perimeter enlargement, an automatic link to the bank accountability in order to accelerate the action task (treatment of the most suspicious operations and release of the less suspicious suspended operations), or simply data skills transfer to fraud experts. Under investment constraints, these pragmatic considerations remind that the "performance" of such AI devices are far from being solely statistical.

\section{Conclusion}

Humans are in the loop in many business processes in banking, as is fraud detection. Just as the task of classifying a tumour is only one aspect of a dermatologist's work, identifying a potential fraud is only one step in a more complex fraud management process. Human processes of evaluation and action against the fraudster makes it difficult to simply compare machine learning algorithms on their performance or explanatory power outside of their use by the practitioner. In the field of fraud detection, although rulebased approaches remain the standard in banks, data-based rule learning approaches can be used to reach much better performance. Furthermore, some tasks of the fraud management can be optimized with black box models, as soon as an explanation of the decision is not required for the investigation or for a legal action against the fraudster. While the question of which approach will give the best answer is still open, we showed on a practical example that it is possible in the meantime to hybridize both approaches to develop improved solutions for the complete fraud management process.

$\begin{array}{ll}\text { Abbreviations } \\ \text { FDM } & \text { fraud detection model } \\ \text { FN } & \text { false negatives } \\ \text { FN } & \text { false negatives } \\ \text { FP } & \text { false positives } \\ \text { FTE } & \text { full time equivalent } \\ \text { SOTA } & \text { state-of-the-art } \\ \text { TN } & \text { true negatives } \\ \text { TP } & \text { true positives }\end{array}$

Acknowledgments. The authors are grateful for the support provided by Quinten, for colleagues' reviews and for the careful rereading of the final English version, as well as for the long collaboration with Bleckwen, ERDF, and our partner Banque Populaire Rives de Paris who shared their data and business expertise. We would also like to thank the Data for Policy organization team for their kind advices for the conference preparation (submission \#35, DOI:10.5281/zenodo.3967821).

Funding Statement. This research was supported by ERDF under research grants, exclusively covering the mobilized human resources, in the framework of a European project to propose innovative solutions for fraud management. The funder had no role in study design, data collection and analysis, decision to publish, or preparation of the manuscript.

Competing Interests. Anna Nesvijevskaia, Sophie Ouillade, and Pauline Guilmin are employed at company Quinten, part of a consortium with Bleckwen who received grant from ERDF. Jean-Daniel Zucker declares none.

Data Availability Statement. The data that support the findings of this study are available from Quinten's partner Banque Populaire Rives de Paris. For reasons of confidentiality, we cannot provide these data.

Author Contributions. Conceptualization, A.N. and J.-D.Z.; Methodology, A.N. and J.-D.Z.; Investigation, A.N., J.-D.Z., S.O., and P.G.; Validation, A.N.; Writing-original draft, A.N., J.-D.Z., S.O., and P.G.; Writing-review \& editing, A.N., J.-D.Z., S.O., and P.G.; Visualization, A.N., S.O., and P.G.; Supervision, A.N.; Funding acquisition, A.N.; Project administration, A.N. and S.O.; Software, S.O. and P.G.; Formal analysis, S.O. and P.G.; Data curation, S.O. and P.G. All authors approved the final submitted draft.

\section{References}

Abdallah A, Maarof MA and Zainal A (2016) Fraud detection system: A survey. Journal of Network and Computer Applications $68,90-113$.

Achituve I, Kraus S and Goldberger J (2019) Interpretable online banking fraud detection based on hierarchical attention mechanism. In 2019 IEEE 29th International Workshop on Machine Learning for Signal Processing (MLSP), pp. 1-6. 
Aggarwal CC (2017) Outlier Analysis, 2nd Edn. Berlin: Springer International Publishing. https://doi.org/10.1007/978-3-31947578-3

Alexopoulos P, Kafentzis K, Benetou X, Tagaris T and Georgolios P (2007) Towards a generic fraud ontology in e-government. ICE B, 269-276.

Amrane M, Civet A, Templier A, Kang D and Figueiredo FC (2015) Patients with moderate to severe dry eye disease in routine clinical practice in the UK - Physician and patient's assessments. Investigative Ophthalmology \& Visual Science 56(7), 4443-4443.

Apley DW and Zhu J (2019) Visualizing the effects of predictor variables in black box supervised learning models. ArXiv: 1612.08468 [Stat]. Available at http://arxiv.org/abs/1612.08468

Arrieta AB, Díaz-Rodríguez N, Del Ser J, Bennetot A, Tabik S, Barbado A, Garcia S, Gil-Lopez S, Molina D, Benjamins R, Chatila R and Herrera F (2020) Explainable artificial intelligence (XAI): Concepts, taxonomies, opportunities and challenges toward responsible AI. Information Fusion 58, 82-115.

Bach S, Binder A, Montavon G, Klauschen F, Müller K-R. and Samek W (2015) On pixel-wise explanations for non-linear classifier decisions by layer-wise relevance propagation. PLOS ONE 10(7), e0130140. https://doi.org/10.1371/journal.pone.0130140

Batista G, Prati R and Monard M-C. (2004) A study of the behavior of several methods for balancing machine learning training data. SIGKDD Explorations 6, 20-29.

Bifet A and Gavaldà R (2007) Learning from time-changing data with adaptive windowing. In Proceedings of the 2007 SIAM International Conference on Data Mining, Society for Industrial and Applied Mathematics, pp. 443-448.

Bissell AF (1986) Corrigendum: The performance of control charts and cusums under linear trend. Journal of the Royal Statistical Society. Series C (Applied Statistics) 35(2), 214-214.

Bottou L, Peters J, Quiñonero-Candela J, Charles DX, Chickering DM, Portugaly E, Ray D, Simard P and Snelson E (2013) Counterfactual reasoning and learning systems. ArXiv:1209.2355 [Cs, Math, Stat]. Available at http://arxiv.org/abs/1209.2355

Breiman L (2001) Random forests. Machine Learning 45(1), 5-32.

Brennan, P. (2012, October 10). A comprehensive survey of methods for overcoming the class imbalance problem in fraud detection, Thesis directed by Hofmann M., Institute of Technology Blanchardstown, Dublin, Ireland.

Breunig M, Kriegel H-P., Ng RT and Sander J (2000) LOF: Identifying density-based local outliers. In Proceedings of the 2000 Acm Sigmod International Conference on Management of Data, ACM, pp. 93-104.

Campello RJGB, Moulavi D, Zimek A and Sander J (2015) Hierarchical density estimates for data clustering, visualization, and outlier detection. ACM Transactions on Knowledge Discovery from Data 10, 1-51.

Candès EJ, Li X, Ma Y and Wright J (2011) Robust principal component analysis? Journal of the ACM 58(3), 1-37.

Chawla NV, Bowyer KW, Hall LO and Kegelmeyer WP (2002) SMOTE: Synthetic minority over-sampling technique. Journal of Artificial Intelligence Research 16, 321-357.

Chawla NV, Lazarevic A, Hall LO and Bowyer KW (2003) SMOTEBoost: Improving prediction of the minority class in boosting. In Proceedings of the Principles of Knowledge Discovery in Databases, PKDD-2003, pp. 107-119.

Choi D and Lee K (2018) An artificial intelligence approach to financial fraud detection under IoT environment: A survey and implementation. Security and Communication Networks. 2018, 15 doi:10.1155/2018/5483472

Dal Pozzolo A, Boracchi G, Caelen O, Alippi C and Bontempi G (2015) Credit card fraud detection and concept-drift adaptation with delayed supervised information. In 2015 International Joint Conference on Neural Networks (IJCNN), pp. 1-8.

Dal Pozzolo A, Caelen O, Le Borgne Y-A, Waterschoot S and Bontempi G (2014) Learned lessons in credit card fraud detection from a practitioner perspective. Expert Systems with Applications 41(10), 4915-4928.

Davis J and Goadrich M (2006) The relationship between precision-recall and ROC curves. Proceedings of the 23rd International Conference on Machine Learning (ICML) 148, 233-240.

Dhankhad S, Mohammed E and Far B (2018). Supervised machine learning algorithms for credit card fraudulent transaction detection: A comparative study. In 2018 IEEE International Conference on Information Reuse and Integration (IRI), pp. $122-125$.

Domingos P (1999) MetaCost: A general method for making classifiers cost-sensitive. In Proceedings of the Fifth ACM SIGKDD International Conference on Knowledge Discovery and Data Mining. New York, NY: Association for Computing Machinery, pp. $155-164$.

Domingos P (2015) The Master Algorithm: How the Quest for the Ultimate Learning Machine Will Remake Our World. London: Penguin.

Domingos P and Hulten G (2000) Mining high-speed data streams. In Proceedings of the Sixth ACM SIGKDD International Conference on Knowledge Discovery and Data Mining. New York, NY: Association for Computing Machinery, pp. 71-80.

Elkan C (2001) The foundations of cost-sensitive learning. In Proceedings of the Seventeenth International Joint Conference on Artificial Intelligence, pp. 973-978.

Fan W, Stolfo SJ, Zhang J and Chan PK (1999) AdaCost: Misclassification cost-sensitive boosting. In Proceedings of the Sixteenth International Conference on Machine Learning. San Francisco, CA: Morgan Kaufmann Publishers Inc., pp. 97-105.

Fiore U, Santis A, Perla F, Zanetti P and Palmieri F (2019) Using generative adversarial networks for improving classification effectiveness in credit card fraud detection. Information Sciences 479, 448-455.

Fisher A, Rudin C and Dominici F (2019) All models are wrong, but many are useful: Learning a variable's importance by studying an entire class of prediction models simultaneously. Journal of Machine Learning Research 20(177), 1-81.

Fridman L (2019) Deep Learning State of the Art (introductory lecture for MIT course 6.S094). Available at https://www.youtube. com/watch?v=0VH1Lim8gL8 (accessed 26 February 2021). 
Friedman JH (2001) Greedy function approximation: A gradient boosting machine. Annals of Statistics 29(5), 1189-1232.

Friedman JH and Popescu BE (2008) Predictive learning via rule ensembles. ArXiv:0811.1679 [Stat]. https://doi.org/10.1214/07-AOAS148

Gama J, Žliobaitė I, Bifet A, Pechenizkiy M and Bouchachia A (2014) A survey on concept drift adaptation. ACM Computing Surveys 46(4), 1-37.

Goldstein A, Kapelner A, Bleich J and Pitkin E (2014) Peeking inside the black box: Visualizing statistical learning with plots of individual conditional expectation. ArXiv:1309.6392 [Stat]. Available at http://arxiv.org/abs/1309.6392

Hart P (1968) The condensed nearest neighbor rule (Corresp.). IEEE Transactions on Information Theory 14(3), 515-516.

Hawkins S, He H, Williams G and Baxter R (2002) Outlier detection using replicator neural networks. In Kambayashi Y, Winiwarter W and Arikawa M (eds), Data Warehousing and Knowledge Discovery. Berlin, Heidelberg: Springer, pp. $170-180$.

He H, Bai Y, Garcia EA and Li S (2008) ADASYN: Adaptive synthetic sampling approach for imbalanced learning. In 2008 IEEE International Joint Conference on Neural Networks (IEEE World Congress on Computational Intelligence), pp. 1322-1328.

He Z, Xu X and Deng S (2003) Discovering cluster-based local outliers. Pattern Recognition Letters 24(9), 1641-1650.

Hinton G, Vinyals $O$ and Dean J (2015) Distilling the knowledge in a neural network. ArXiv:1503.02531 [Cs, Stat]. Available at http://arxiv.org/abs/1503.02531

Hooker G (2004) Discovering additive structure in black box functions. In Proceedings of the Tenth ACM SIGKDD International Conference on Knowledge Discovery and Data Mining, New York, NY: Association for Computing Machinery, pp. 575-580.

Jakulin A, Možina M, Demšar J, Bratko I and Zupan B (2005) Nomograms for visualizing support vector machines. In Proceedings of the Eleventh ACM SIGKDD International Conference on Knowledge Discovery in Data Mining. New York, NY: Association for Computing Machinery, pp. 108-117.

Knorr E and Ng R (1998) Algorithms for mining distance-based outliers in large datasets. VLDB.

Kou Y, Lu C-T, Sirwongwattana S and Huang Y-P (2004) Survey of fraud detection techniques. In IEEE International Conference on Networking, Sensing and Control, 2004 2, 749-754.

Kubat M and Matwin S (1997) Addressing the curse of imbalanced training sets: One-sided selection. In Proceedings of the Fourteenth International Conference on Machine Learning. Burlington, MA: Morgan Kaufmann, pp. 179-186.

Lau N, Fridman L, Borghetti B and Lee J (2018) Machine learning and human factors: Status, applications, and future directions. Proceedings of the Human Factors and Ergonomics Society Annual Meeting 62, 135-138.

Laurikkala J (2001) Improving identification of difficult small classes by balancing class distribution. In Quaglini S, Barahona P and Andreassen S (eds), Artificial Intelligence in Medicine. Berlin, Heidelberg: Springer, pp. 63-66.

Liu FT, Ting KM and Zhou Z-H. (2012) Isolation-based anomaly detection. ACM Transactions on Knowledge Discovery from Data 6(1), 3:1-3:39.

Lu J, Liu A, Dong F, Gu F, Gama J and Zhang G (2019) Learning under concept drift: A review. IEEE Transactions on Knowledge and Data Engineering 31(12), 2346-2363.

Lundberg SM, Erion GG and Lee S-I (2019) Consistent Individualized Feature Attribution for Tree Ensembles. ArXiv: 1802.03888 [Cs, Stat]. Available at http://arxiv.org/abs/1802.03888

Lundberg SM and Lee S-I (2017) A unified approach to interpreting model predictions. Advances in Neural Information Processing Systems 30, 4765-4774.

Morde V (2019) XGBoost Algorithm: Long May She Reign! Available at https:/towardsdatascience.com/https-medium-comvishalmorde-xgboost-algorithm-long-she-may-rein-edd9f99be63d (accessed 26 February 2021).

Murdoch WJ, Singh C, Kumbier K, Abbasi-Asl R and Yu B (2019) Definitions, methods, and applications in interpretable machine learning. Proceedings of the National Academy of Sciences 116(44), 22071-22080.

Nesvijevskaia A (2021) Databook: Standardised framework for dynamic documentation of algorithm design during data science projects. IASSIST Quarterly 45, 34.

Ramaswamy S, Rastogi R and Shim K (2000) Efficient algorithms for mining outliers from large data sets. $A C M S I G M O D 29(2)$, 427-438. https://doi.org/10.1145/335191.335437

Rao JS and Potts WJE (1997) Visualizing bagged decision trees. KDD, pp. 243-246.

Ribeiro MT, Singh S and Guestrin C (2016) "Why Should I Trust You?": Explaining the predictions of any classifier. In Proceedings of the 22nd ACM SIGKDD International Conference on Knowledge Discovery and Data Mining. New York, NY: Association for Computing Machinery, pp. 1135-1144.

Roberts SW (1966) A comparison of some control chart procedures. Technometrics 8(3), 411-430.

Rousseeuw P and Driessen K (1999) A fast algorithm for the minimum covariance determinant estimator. Technometrics 41, 212-223.

Rudin C (2019) Stop explaining black box machine learning models for high stakes decisions and use interpretable models instead. Nature Machine Intelligence 1(5), 206-215.

Ryman-Tubb NF, Krause P and Garn W (2018) How artificial intelligence and machine learning research impacts payment card fraud detection: A survey and industry benchmark. Engineering Applications of Artificial Intelligence 76, $130-157$.

Sadgali I, Sael N and Benabbou F (2019) Performance of machine learning techniques in the detection of financial frauds Procedia Computer Science 148, 45-54.

Sahin EK (2020) Assessing the predictive capability of ensemble tree methods for landslide susceptibility mapping using XGBoost, gradient boosting machine, and random forest. SN Applied Sciences 2(7), 1308. 
Sahin Y, Bulkan S and Duman E (2013) A cost-sensitive decision tree approach for fraud detection. Expert Systems with Applications 40, 5916-5923.

Saito T and Rehmsmeier M (2015) The precision-recall plot is more informative than the ROC plot when evaluating binary classifiers on imbalanced datasets. PLOS ONE 10(3), e0118432. https://doi.org/10.1371/journal.pone.0118432

SamanehSorournejad, Zojaji Z, Atani RE and Monadjemi AH (2016) A survey of credit card fraud detection techniques: Data and technique oriented perspective. ArXiv:1611.06439 [Cs]. Available at http://arxiv.org/abs/1611.06439

Schölkopf B, Platt JC, Shawe-Taylor J, Smola AJ and Williamson RC (2001) Estimating the support of a high-dimensional distribution. Neural Computation 13(7), 1443-1471.

Shah V, Shah P, Shetty $\mathbf{H}$ and Mistry K (2019) Review of credit card fraud detection techniques. In 2019 IEEE International Conference on System, Computation, Automation and Networking (ICSCAN), pp. 1-7.

Shearer C (2000) The CRISP-DM model : The new blueprint for data mining. Journal of Data Warehousing 5, 13-22.

Shehnepoor S, Togneri R, Liu W and Bennamoun M (2020) GANgster: A fraud review detector based on regulated GAN with data augmentation. ArXiv:2006.06561 [Cs, Stat]. Available at http://arxiv.org/abs/2006.06561

Shiryaev AN (1961) The problem of the most rapid detection of a disturbance in a stationary process. Soviet Mathematics - Doklady 2, 795-799.

Shrikumar A, Greenside P and Kundaje A (2017) Learning important features through propagating activation differences. Proceedings of the 34th International Conference on Machine Learning 70, 3145-3153.

Shukla PK and Tripathi SP (2011) A survey on interpretability-accuracy (I-A) trade-off in evolutionary fuzzy systems. In 2011 Fifth International Conference on Genetic and Evolutionary Computing, pp. 97-101.

Tang J, Chen Z, Fu AW and Cheung DW (2002) Enhancing effectiveness of outlier detections for low density patterns. In Chen M-S, Yu PS and Liu B (eds), Advances in Knowledge Discovery and Data Mining. Berlin, Heidelberg: Springer, pp. 535-548.

Tomek I (1976) Two modifications of CNN. IEEE Transactions on Systems, Man, and Cybernetics, 6(11), 769-772.

Tsang M, Sun Y, Ren D and Liu Y (2018) Can I trust you more? Model-agnostic hierarchical explanations. ArXiv:1812.04801 [Cs, Stat]. Available at http://arxiv.org/abs/1812.04801

Tsymbal, A. (2004). The Problem of Concept Drift: Definitions and Related Work. Available at https://www-ai.cs.tu-dortmund.de/ LEHRE/FACHPROJEKT/SS12/paper/concept-drift/tsymbal2004.pdf

Wachter S, Mittelstadt B and Russell C (2018) Counterfactual explanations without opening the black box: Automated decisions and the GDPR. ArXiv:1711.00399 [Cs]. Available at http://arxiv.org/abs/1711.00399

Walke A (2019) Comparison of supervised and unsupervised fraud detection. In Alfaries A, Mengash H, Yasar A and Shakshuki E (eds), Advances in Data Science, Cyber Security and IT Applications. Cham: Springer International Publishing, pp. 8-14.

Webb GI, Hyde R, Cao H, Nguyen HL and Petitjean F (2016) Characterizing concept drift. Data Mining and Knowledge Discovery 30(4), 964-994.

Weerts H, Ipenburg W and Pechenizkiy M (2019) Case-Based Reasoning for Assisting Domain Experts in Processing Fraud Alerts of Black-Box Machine Learning Models. Available at https://arxiv.org/pdf/1907.03334.pdf

Widmer G and Kubat M (1994) Learning in the presence of concept drift and hidden contexts. Machine Learning 23, 69-101. https://doi.org/10.1007/BF00116900

Wilhelm WK (2003) The Fraud Management Lifecycle Theory: A Holistic Approach to Fraud Management. Utica, NY: Utica College.

Wilson DL (1972) Asymptotic properties of nearest neighbor rules using edited data. IEEE Transactions on Systems, Man, and Cybernetics 2(3), 408-421.

Wirth R and Hipp J (2000) CRISP-DM: Towards a standard process model for data mining. In Proceedings of the Fourth International Conference on the Practical Application of Knowledge Discovery and Data Mining, pp. 29-39.

Yang YJ and Bang CS (2019) Application of artificial intelligence in gastroenterology. World Journal of Gastroenterology 25(14), 1666-1683.

Yu B and Kumbier K (2020) Veridical data science. Proceedings of the National Academy of Sciences of the United States of America 117, 3920-3929. https://doi.org/10.1073/pnas.1901326117

Zhou FL, Watada H, Tajima Y, Berthelot M, Kang D, Esnault C, Shuto Y, Maegawa H and Koya D (2019) Identification of subgroups of patients with type 2 diabetes with differences in renal function preservation, comparing patients receiving sodiumglucose co-transporter- 2 inhibitors with those receiving dipeptidyl peptidase- 4 inhibitors, using a supervised machine-learning algorithm (PROFILE study): A retrospective analysis of a Japanese commercial medical database. Diabetes, Obesity and Metabolism 21(8), 1925-1934.

Zhuang H, Wang X, Bendersky M, Grushetsky A, Wu Y, Mitrichev P, Sterling E, Bell N, Ravina W and Qian H (2020) Interpretable learning-to-rank with generalized additive models. ArXiv:2005.02553 [Cs, Stat]. Available at http://arxiv.org/abs/2005.02553

Žliobaitė I (2010) Learning under concept drift: An overview. ArXiv:1010.4784 [Cs]. Available at http://arxiv.org/abs/1010.4784

Žliobaitė I, Pechenizkiy M and Gama J (2016) An overview of concept drift applications. In Japkowicz N and Stefanowski J (eds), Big Data Analysis: New Algorithms for a New Society. Cham: Springer International Publishing, pp. 91-114.

Cite this article: Nesvijevskaia A, Ouillade S, Guilmin P and Zucker J.-D (2021). The accuracy versus interpretability trade-off in fraud detection model. Data \& Policy, 3: e12. doi:10.1017/dap.2021.3 


\section{Appendix}

Table A1. Solutions to deal with imbalanced data.

\begin{tabular}{|c|c|c|c|c|}
\hline Name & $\begin{array}{l}\text { Data or } \\
\text { algorithm } \\
\text { modification }\end{array}$ & $\begin{array}{l}\text { Under or over } \\
\text { sampling }\end{array}$ & Advantages & Drawbacks \\
\hline $\begin{array}{l}\text { Random over- } \\
\text { sampling }\end{array}$ & Data & Over-sampling & $\begin{array}{l}\text { - Easy to implement } \\
\text { - Generates observations with real character- } \\
\text { istics of the positive class (as duplicated } \\
\text { observations) }\end{array}$ & $\begin{array}{l}\text { - Can induce over-fitting to specific } \\
\text { patterns of fraud } \\
\text { - Insufficient in the case of extremely } \\
\text { imbalanced data }\end{array}$ \\
\hline SMOTE & Data & Over-sampling & $\begin{array}{l}\text { - Does not duplicate existent observations } \\
\text { - Generates relevant observations }\end{array}$ & $\begin{array}{l}\text { - In case of overlapped classes (cre- } \\
\text { ates fallacious observations) }\end{array}$ \\
\hline SMOTEBoost & Data & Over-sampling & $\begin{array}{l}\text { - Does not duplicate existent observations } \\
\text { - Generates relevant observations } \\
\text { - Rebalanced on misclassified observations }\end{array}$ & $\begin{array}{l}\text { - In case of overlapped classes (cre- } \\
\text { ates fallacious observations) }\end{array}$ \\
\hline ADASYN & Data & Over-sampling & $\begin{array}{l}\text { - Does not duplicate existent observations } \\
\text { - Generates relevant observations } \\
\text { - Takes into account the local neighborhood: } \\
\text { less fallacious observations created }\end{array}$ & $\begin{array}{l}\text { - In case of overlapped classes (cre- } \\
\text { ates fallacious observations) }\end{array}$ \\
\hline GAN & Data & Over-sampling & $\begin{array}{l}\text { - Generates extremely relevant information } \\
\text { - Could perform well in case of overlapped } \\
\text { classes }\end{array}$ & $\begin{array}{l}\text { - Needs a large number of fraudulent } \\
\text { observations to be trained }\end{array}$ \\
\hline $\begin{array}{l}\text { Random } \\
\text { under- } \\
\text { sampling }\end{array}$ & Data & Under-sampling & $\begin{array}{l}\text { - Easy to implement } \\
\text { - Can be used to suppress redundant or useless } \\
\text { observations } \\
\text { - Does not suppress } 1 \text { group of observations } \\
\text { but disperse observations }\end{array}$ & $\begin{array}{l}\text { - Can suppress relevant observations } \\
\text { - Insufficient in the case of extremely } \\
\text { imbalanced data }\end{array}$ \\
\hline $\begin{array}{l}\text { Near miss or } \\
\text { CNN }\end{array}$ & Data & $\begin{array}{l}\text { Under-sampling } \\
\text { Selects }\end{array}$ & $\begin{array}{l}\text { - Keeps the observations at the border (hard to } \\
\text { separate) }\end{array}$ & $\begin{array}{l}\text { Highly probable to suppress rele- } \\
\text { vant observations: drastically sup- } \\
\text { pression }\end{array}$ \\
\hline
\end{tabular}




\begin{tabular}{|c|c|c|c|c|}
\hline Name & $\begin{array}{l}\text { Data or } \\
\text { algorithm } \\
\text { modification }\end{array}$ & $\begin{array}{l}\text { Under or over } \\
\text { sampling }\end{array}$ & Advantages & Drawbacks \\
\hline & & $\begin{array}{l}\text { observations to } \\
\text { keep }\end{array}$ & $\begin{array}{l}\text { - Suppresses observations that could be use- } \\
\text { less }\end{array}$ & $\begin{array}{l}\text { Makes harder the separation of } \\
\text { classes: more difficult for the model } \\
\text { to perform }\end{array}$ \\
\hline $\begin{array}{l}\text { Tomek links } \\
\text { or ENN }\end{array}$ & Data & $\begin{array}{l}\text { Under-sampling } \\
\text { Selects } \\
\text { observations to } \\
\text { remove }\end{array}$ & $\begin{array}{l}\text { - Simplifies the problem at the border } \\
\text { - Better to describe cluster a posterior }\end{array}$ & $\begin{array}{l}\text { - Illusion that the problem is easier } \\
\text { than it is } \\
\text { - Insufficient in the case of extremely } \\
\text { imbalanced data }\end{array}$ \\
\hline OSS or NCL & Data & $\begin{array}{l}\text { Under-sampling } \\
\text { Selects } \\
\text { observations to } \\
\text { keep and to remove }\end{array}$ & $\begin{array}{l}\text { - Combines solutions } \\
\text { - More precisely removes data }\end{array}$ & - Difficult to parameterize \\
\hline SMOTE-ENN & Data & $\begin{array}{l}\text { Hybrid (Under and } \\
\text { over-sampling) }\end{array}$ & $\begin{array}{l}\text { - Can be sufficient with extremely imbalanced } \\
\text { data }\end{array}$ & $\begin{array}{l}\text { - Difficult to parameterize simulta- } \\
\text { neously over and under sampling }\end{array}$ \\
\hline $\begin{array}{l}\text { SMOTE } \\
\text { Tomek }\end{array}$ & Data & $\begin{array}{l}\text { Hybrid (Under and } \\
\text { over-sampling) }\end{array}$ & $\begin{array}{l}\text { - Can be sufficient with extremely imbalanced } \\
\text { data }\end{array}$ & $\begin{array}{l}\text { Difficult to parameterize simulta- } \\
\text { neously over and under sampling }\end{array}$ \\
\hline $\begin{array}{l}\text { Cost function: } \\
\text { adding } \\
\text { weights }\end{array}$ & Algorithm & NA & $\begin{array}{l}\text { - Easy to implement (already exists in built-in } \\
\text { sklearn model) } \\
\text { - Improves algorithm performance }\end{array}$ & $\begin{array}{l}\text { - Does not distinguish observations } \\
\text { easy or hard to learn or } \\
\text { - Hard to choose an arbitrary weight } \\
\text { - Insufficient in the case of extremely } \\
\text { imbalanced data }\end{array}$ \\
\hline $\begin{array}{l}\text { AdaCost/ } \\
\text { MetaCost }\end{array}$ & Algorithm & NA & $\begin{array}{l}\text { - Over-sampling within the algorithm } \\
\text { - Gives more importance to positive observa- } \\
\text { tions hard to learn }\end{array}$ & $\begin{array}{l}\text { Can be hard to implement (not } \\
\text { available in built-in function) }\end{array}$ \\
\hline
\end{tabular}

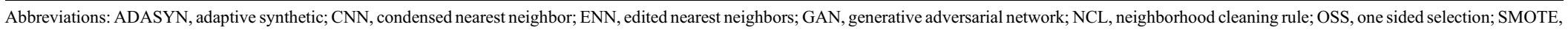
synthetic minority over-sampling technique. 
Table A2. Methods used for anomaly detection.

\begin{tabular}{|c|c|c|c|c|}
\hline Name & $\begin{array}{l}\text { Unsupervised or semi- } \\
\text { supervised }\end{array}$ & $\begin{array}{l}\text { Distance or density } \\
\text { based }\end{array}$ & Advantages & Drawbacks \\
\hline$Z$-score & Unsupervised & Distance based & $\begin{array}{l}\text { - Simple method } \\
\text { - Easy to visualize results } \\
\text { - No parameterization }\end{array}$ & $\begin{array}{l}\text { - Global approach: depending on } \\
\text { the calculated mean of data } \\
\text { - May not work in case of over- } \\
\text { lapped classes }\end{array}$ \\
\hline $\begin{array}{l}\mathrm{k}-\mathrm{NN} \\
\quad \text { distance }\end{array}$ & Unsupervised & Distance based & $\begin{array}{l}\text { - Simple method } \\
\text { - Easy to visualize result } \\
\text { - Not a global approach }\end{array}$ & $\begin{array}{l}\text { - Difficult to parameterize } \\
\text { - May not work in case of over- } \\
\text { lapped classes }\end{array}$ \\
\hline LOF & Unsupervised & Density based & $\begin{array}{l}\text { - Local approach } \\
\text { - Takes density of the neighborhood into } \\
\text { account: more relevant in many cases } \\
\text { - Possible to use other distance metrics }\end{array}$ & $\begin{array}{l}\text { - Difficult to parameterize } \\
\text { - Not easily interpretable } \\
\text { - May not work in case of over- } \\
\text { lapped classes }\end{array}$ \\
\hline $\mathrm{COF}$ & Unsupervised & Density based & $\begin{array}{l}\text { - Local approach } \\
\text { - Understands complex forms of neigh- } \\
\text { borhoods }\end{array}$ & $\begin{array}{l}\text { - Difficult to parameterize } \\
\text { - Not easily interpretable } \\
\text { - May not work in case of over- } \\
\text { lapped classes }\end{array}$ \\
\hline $\begin{array}{l}\text { Isolation } \\
\text { forest }\end{array}$ & Unsupervised & Density based & $\begin{array}{l}\text { - Easily interpretable } \\
\text { - Takes into account both density and } \\
\text { distance }\end{array}$ & $\begin{array}{l}\text { - May not be sufficiently local: } \\
\text { comparison to the average deep } \\
\text { - Hard to parameterize }\end{array}$ \\
\hline CBLOF & Unsupervised & Cluster based & $\begin{array}{l}\text { - Local approach } \\
\text { - Takes into account both density and } \\
\text { distance }\end{array}$ & $\begin{array}{l}\text { - Difficult to parameterize } \\
\text { - May not work in case of over- } \\
\text { lapped classes }\end{array}$ \\
\hline DBSCAN & Unsupervised & Cluster based & - Local approach & - Hard to parameterize \\
\hline
\end{tabular}


Table A2. Continued

\begin{tabular}{|c|c|c|c|c|}
\hline Name & $\begin{array}{l}\text { Unsupervised or semi- } \\
\text { supervised }\end{array}$ & $\begin{array}{l}\text { Distance or density } \\
\text { based }\end{array}$ & Advantages & Drawbacks \\
\hline & & & $\begin{array}{l}\text { - Clusters the data in addition with } \\
\text { detecting anomalous observations } \\
\text { - Takes into account both density and } \\
\text { distance }\end{array}$ & $\begin{array}{l}\text { May not work in case of over- } \\
\text { lapped classes }\end{array}$ \\
\hline r-PCA & Unsupervised & Distance based & $\begin{array}{l}\text { - Reduces influence of collinearity } \\
\text { between variables } \\
\text { - Reduces influence of noise }\end{array}$ & $\begin{array}{l}\text { - High computational complexity: } \\
\text { may not work on large data } \\
\text { - May not work in case of over- } \\
\text { lapped classes }\end{array}$ \\
\hline $\begin{array}{l}\text { One class } \\
\text { SVM }\end{array}$ & Semi-supervised & NA & $\begin{array}{l}\text { - Simple methods } \\
\text { - Easy to visualize results }\end{array}$ & $\begin{array}{l}\text { - May not be sufficiently local } \\
\text { - May not work in case of over- } \\
\text { lapped classes }\end{array}$ \\
\hline $\begin{array}{l}\text { Auto } \\
\text { encoder }\end{array}$ & Semi-supervised & NA & $\begin{array}{l}\text { - No need to create derived variables } \\
\text { - Precise results: excellent performance } \\
\text { - Handles overlapped classes }\end{array}$ & $\begin{array}{l}\text { - Needs a large dataset as input } \\
\text { - Not easily interpretable }\end{array}$ \\
\hline
\end{tabular}

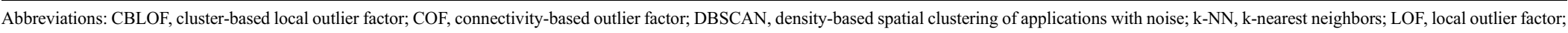
r-PCA, robust principal component analysis; SVM, support vector machines. 